\title{
Complications and Management of Triplet Pregnancv
}

Vaidva $A$.

\begin{abstract}
Triblet pregnancies are associated with an increased risk of maternal and neonatal complications. Preterm deliverv is the most significant complication of triplet gestation. The maior cause of increased incidence of triple pregnancies is the increasing use of fertilitv treatments. Common complications include preeclampsia. preterm premature rupture of membrane. fetal growth restriction an non- reassuring fetal cardiotocograph tracing.

Despite the improving survival of triplets. effective measure to prevent significant proportion of potential neurological morbiditv in triplet babies should be taken into account
\end{abstract}

Kevwords: Pregnancv. preterm. triplet

\section{Introduction}

Triplet pregnancies are associated with an increased risk of maternal and neonatal complications. Preterm deliverv is the most significant complication of triplet gestation: 75 to $100 \%$ of triblets are born prematurelv. The maior cause of increased incidence of pregnancies is the increasing use fertilitv treatments. Common etiologies include preeclampsia. preterm premature rupture of membrane. fetal growth restriction and non-reassuring fetal heart tracing.

Antenartum interventions for prevention of preterm birth Bed rest and hospitalization: Bed rest is the traditional management for manv obstetrical complications. Retrospective analvses have not clearlv demonstrated the benefit of bed rest for prevention of preterm deliverv. There is no consensus regarding bed rest and hospitalization for women carrving triplets.

Cerclage : The elective use of cervical cerclage in triplet pregnancv is not recommended because supporting data is not vet available.

Prophvlactic tocolvsis : There are no randomized controlled trails of prophvlactic tocolvsis

Cervical examination: Most protocols for the outpatient management of triplets include digital examination of the cervix on a weeklv basis starting at 20 to 24 weeks of gestation. Detection of cervical change should lead to evaluation for occult contractions and tocolvtic therapv. if appropriate. No intervention has been shown to improve outcome when cervical change is not accompanied bv uterine contractions.
Antenatal corticosteroids: It is difficult to point out a time to start antenatal corticosteroid administration for fetus at risk for preterm birth because of increasing evidence suggestive of potential ill effects from multiple courses of steroids. So administration is to be considered until there is clear evidence of preterm labor

Ultrasound assessment of cervical length: The mean cervical length in case of triblet gestations is less than that of singletons ${ }^{2}$. In one studv. triplet gestation delivering prior to 33 weeks had shorter cervices at 28. $30 \& 32$ weeks than those delivering after 33 weeks ${ }^{2}$. It has been suggested that a cervical length less than $25 \mathrm{~mm}$ at 23 to 24 weeks of gestation indicates pregnancies at highest risk. Sonographv mav help to identifv patients at high risk for preterm deliverv.

Eclambsia : Preeclampsia comblicates 20 to 46 percent of triplet gestations compared to 5 percent of singleton pregnancies ${ }^{3}$. Prenatal visits are scheduled more freauentlv for women carrving triplets. to monitor for the development of hvpertension and proteinuria.

Preterm premature rupture of membranes: PPROM is another cause of prematuritv. In reviewing the literature. the incidence of this complication appeared to be approximatelv 15 to 30 percent in three series including 300 triplet pregnancies ${ }^{4}$.

Fetal growth restriction: In one series of 57 triplet pregnancies. severe growth restriction was listed as the primarv indication for deliverv in 3.5 percent $^{5}$.

Corresponding Author: Vaidva A.. E-mail: achala vaidva@hotmail.com: Maturnitv Hospital. Thapathali. kathmandu. Nepal. 
Antepartum monitoring: Weeklv fetal surveillance with nonstress tests or biophvsical profiles is generallv initiated at $32 \mathrm{weeks}^{6}$. But in the presence of intrauterine growth restriction. it should be started earlier.

Fetal growth: Intrauterine growth restriction and related complications (eg. oligohvdramnios. nonreassuring fetal heart rate) are more common in triblets than in singletons ${ }^{7}$. For this reason. most management protocols include serial sonography for evaluation of fetal growth everv three to four weeks. It is likelv that disparitv in growth reflects placental dvsfunction ${ }^{8}$. Therefore. sonographic evidence of significant fetal weight discordance warrants close fetal monitoring and possible earlv deliverv.

Velamentous insertion of the umbilical cord is common in triplet gestations. Furthermore. a triplet perfused via a cord with a velamentous insertion is at increased risk for poor fetal growth ${ }^{9}$.

Cholestasis of pregnancv: Intrahepatic cholestasis is observed more commonlv in multiple gestations. Management includes supbortive care for the mother and antepartum fetal monitoring because of the increased risk of stillbirth.

Gestational Anemia : Anemia has been reported in 20 to 70 percent. Iron reauirements for triplets have not been determined. The current recommendation for singleton pregnancv is to increase iron consumption bv $15 \mathrm{mg} / \mathrm{dav}$.

Gestational diabetes : The prevalence of gestational diabetes mellitus appears increased in triplet compared to singleton pregnancies. although this has not been a consistent finding in all studies ${ }^{10}$.

Problems uniaue to multiple gestation: Premature deliverv or death of one fetus with surviving co-fetuses in-utero are complications unique to multiple gestation.

Intervention for one compromised fetus : In multiple gestations. an ethical issue arises when one fetus is in ieopardv. but the others are not. Deliverv before 26 weeks of gestation places all the three fetuses at risk of mortalitv. Therefore. intervention prior to 26 weeks should rarelv be considered ${ }^{11}$. The risk of morbiditv at or bevond 32 weeks of gestation is low. So intervention is nearlv alwavs appropriate. Hence decision should be made after counseling the parents.

Late death of one triplet: Death of one fetus mav increase the risk of morbiditv and mortalitv in a surviving co-triblet. The degree of risk is not well defined but appears to be limited to fetuses with monochorionic placentation. as in twin pregnancies ${ }^{12}$. A studv of 5356 triplet pregnancies found that survival of the remaining triplets after the death of one was inverselv related to the time the death occurred $^{13}$. Mortalitv was higher when the demise occurred later in gestation. Deliverv is recommended when demise is noted and the pregnancv is at least 37 weeks of gestation.

If the pregnancv is trichorionic or dicohorionic with the demised triplet being the one not sharing its chorion. there is no reason to deliver the other triplets. For case of a demised triblet that is one of a monochorionic pair. deliverv is probablv appropriate close to term since potential harmful effects to the survivor likelv occur from hvpotension at the time of the demise ${ }^{14}$. For gestations at 32 weeks or bevond. administering steroids to enhance fetal maturitv and deliverv 48 hours later is an option: however. it is not clear whether this is beneficial to the survivor. as it is possible that the damage occurs to the healthv twin/ triplet less than 48 hours after the demise of the other one. Below 32 weeks. it is probablv best to allow the pregnancy to continue.

Deliverv: A recent large epidemiologic analvsis found that onlv 16 percent of triplets remain undelivered at 36 weeks of gestation ${ }^{15}$. This studv also noted that the prospective risk of fetal death began to exceed neonatal mortalitv at 36 weeks of gestation. So deliverv is considered at this time.

Route of deliverv: The maioritv of triplet fetus is delivered bv cesarean section. This approach is largelv based unon historical data suggesting an increased risk to the fetus from vaginal deliverv. However. this concept has recentlv been challenged $^{16}$. A retrospective series compared the perinatal outcome of 30 triplet pregnancies with planned abdominal deliverv ( 24 actuallv delivered bv cesarean section) to 39 triplet gestations planning for vaginal birth (34 actuallv delivered vaginallv) (16). Planned abdominal deliverv was associated with higher perinatal mortality and neonatal complication rates. primarilv due to more respiratorv distress svndrome (16). Three other studies reported eauivalent neonatal outcomes with vaginal and abdominal birth ${ }^{17}$. Vaginal deliverv mav be safelv offered to a selected population of triplet pregnancies. although the maioritv will require cesarean birth for the usual obstetrical indications (eg. malpresentation. nonreassuring fetal heart rate testing. and extreme prematuritv with an unfavorable cervix). The minimal criteria for allowing a trial of labor are :

- Vertex presentation of the presenting $\left(1^{\text {st }}\right)$ triplet 
- Abilitv to continuouslv monitor all three triplets during labor

- Lack of anv contraindications to vaginal deliverv.

These three criteria are based unon the published studies. which generallv did not put limits on either gestational age or fetal weight.

Techniaue: Vaginal deliverv should onlv be attempted bv experienced who can perform intrauterine manipulation. and operative deliverv. All fetuses should be monitored continuouslv prior to their deliverv due to the possibilitv of nonreassuring fetal heart rates from abruntion or cord prolanse after the birth of the first or second infant. An anesthesiologist should be present at the time of deliverv and prepared for an emergencv cesarean birth or anv manipulation. The deliverv should be performed onlv in places where cesarean deliverv is possible.

The four most common presentations of triplet pregnancv are vertex-breech-breech (18 percent). vertex- vertex-breech (16 percent). Vertex- vertexvertex (15 percent). and breech-breech-breech (13 percent).

Following the vertex deliverv of the first triplet. breech extraction can be performed for rest of the two fetuses pesenting as breech. This reduces the possibilitv of complications.

Cesarean deliverv mav be apvroached bv either a transverse or longitudinal uterine incision ${ }^{18}$. The incision should be adequate to allow atraumatic deliverv of the infants.

Postbartum hemorrhage: The incidence of postnartum hemorrhage after a triplet deliverv is 10 to 35 percent $^{19}$. Thus. one must be readv to manage this problem.

Infant outcome : The infant mortalitv rate of triplets born in the United States from 1995 to 1997 was approximatelv 71 per 1000 births. which was higher than the rate for twins or singletons ( 32 and 6.6 per 1000 births. respectivelv $)^{20}$. The maior reason for this higher rate is the large proportion of LBW. and especiallv VLBW. However. more recent studies have shown improvements in triblet outcomes ${ }_{21}$. One series of 51 triplet gestations reported 96 percent survival at discharge ${ }^{22}$. Triplet infants. have similar outcomes as twins and singletons when stratified bv gestational age ${ }^{23}$. The risk of death is not affected bv birth order ${ }^{24}$.
These tinv neonates often develop significant problems related to their prematuritv. Long-term risk includes cerebral palsv ${ }^{25}$.

\section{Conclusion}

Despite the improving survival of triblets. effective measures to prevent a significant proportion of potential neurological morbiditv in triplet babies should be taken in to account.

\section{Reference:}

1. Newman RB. Hamer C. Miller MC. Outpatient triblet management: a contemporarv review. Am J Obstet Gvnecol. 1989 Sep:161(3):547-53: discussion 553-5.

2. Ramin KD. Ogburn PL Jr. Mulholland TA. Breckle RJ. Ramsev PS. Ultrasonographic assessment of cervical length in triplet pregnancies. Am J Obstet Gvnecol 1999 Jun:180(6 Pt 1):1442-5.

3. Adams DM. Sholl JS. Hanev EI. Russell TL. Silver RK. Perinatal outcome associated with outpatient management of triplet pregnancv. Am J Obstet Gvnecol 1998 Apr:178(4):843-7.

4. Crowther. CA. Hospitalisation and bed rest for multiple pregnancv. In:the Cochrane Librarv. Undate Software. Oxford 2001.

5. Alberecht JL. Tomich PG. The maternal neonatal outcome of triblet gestation. Am J Obstet Gvnecol 1996:174:1551.

6. Newman RB. Ellings JM. Antepartum management of the multiple gestation:the case for specialized care. Semin Perinatol 1995:19:387.

7. Rodis JF. Arkv L. Egan JF. et al.Comprehensive fetal ultrasonogravhic growth measurements in triplet gestation. Am J Obstet Gvnecol 1999:181:1128.

8. Blckstein I. Jacaues DL. Keith LG. A novel approach to intertriplet birth weight discordance. Am J Obstet Gvnecol 2003:188:1926.

9. Feldman DM. Borgida AF. Trvmbulak WP. et al. Clinical implication of Velamentous cord insertion in triplet gestations. Am J Obstet Gvnecol 2002:186:809.

10. Pregnancies and births resulting from in vitro fertilization: French national registrv. analvsis of data 1986 to 1990 . FIVNAT (French In Vitro National). Fertil Steril 1995 Oct:64(4):746-56.

11. Konnikova L. Harvev-Wilkes K. Marion T. et al. Clinical dilemma in triblet pregnancv: when is it appropriate to intervene for a ieopardisid fetus? J Perinatol 2003:23:229.

12. Borlum. KG. Third - trimester fetal death in triplet pregnancies. Obstet Gvnecol 1991:77:6. Journal 
of Nepal Health Research Council Vol.5 No.2 October 2007.

13. Johnson CD. Zhang J. Survival of other fetuses after a fetal death in twin or triplet pregnancies. Obstet Gvvnecol 2002:99:698.

14. Benirschke K. Intrauterine death of a twin: mechanisms. implications for surviving twin. and vlacental pathologv.SeminDiagn Pathol 1993:10:222.

15. Kahn B. Lumev LH. Zvbert OA. et al. Prospective risk of fetal death in singleton. twin. and triplet gestations: Inlications for practice. Obbstet Gvnecol 2003:102:685.

16. Wildschut HI. van Roosmalen J. van Leeuwen E. Keirse MJ. Planned abdominal compared with planned vaginal birth in triplet pregnancies. Br J Obstet Gvnaecol 1995 Apr:102(4):292-6.

17. Grobman WA. Peaceman AM. Hanev EI. et al. Neonatal outcomes in triplet gestations after a trial of labor. Am J Obstet Gvnecol 1998:179:942.

18. Mordel N. Ezra Y. Benshushan A. Dorembus D. Schenker JG. Sadovskv E. Transverse versus longitudinal uterine incision in cesarean deliverv of triplets. J Reprod Med 1993 Sen:38(9):695-6.

19. Malone FD. Kaufman GE. Chelmow D. et al. Maternal morbiditv associated with triplet pregnancv. Am J Perinatol 1998:15:73.
20. Centers for Disease Control and Prevention (CDC). Contribution of assisted reproductive technologv and ovulation-inducing drugs to triplet and higher-order multiole births - United States. 1980-1997. MMWR Morb Mortal Wklv Rep. 2000 Jun 23:49(24):535-8.

21. Maavan Metzge A. Naor N. Sirota. L. Conmparative outcome studv between triplet and singleton preterm newborns. Acta Paediatr 2002:91:1208.

22. Barr S. poggi S. Keszler M. Triplet morbiditv and mortalitv in a large case series $\mathrm{J}$ Perinatol 2003:23:368.

23. Kaufman GE. Malone FD. Harvev-Wilkes KB. et al. Neonatal morbiditv and mortalitv associated with triplet pregnancv. Obstet Gvnecol 1998:91:342.

24. Haloob RK. Kalaivani R. Bagtharia S. Comparison of morbiditv among twins and triplets. J Obstet Gvnecol 2003:23:367.

25. Keith LG. Oleszczuk. JJ. Keith DM. Multiple gestation:reflection on epidemiologv. causes. and conseauences. Int i Fertil Womens Med 2000:45:206. 